783

\section{INTRACRANIAL HEMORRHAGES AND LATE HEMORRHAGIC DISEASE ASSOCIATED CHOLESTATIC LIVER DISEASE}

\author{
H. Per ${ }^{1}$, D. Arslan², H. Gumus ${ }^{1}$, A. Coşkun ${ }^{3}$, \\ S. Kumandas ${ }^{1}$
}

${ }^{1}$ Pediatric Neurology, ${ }^{2}$ Pediatric Gastroenterology, ${ }^{3}$ Pediatric Radiology, Erciyes University, Kayseri, Turkey

Late hemorrhagic disease of the newborn (HDN) is characterized intracranial bleeding in infants aged 1 week-6 month due to severe vitamin $\mathrm{K}$ deficiency, occurring particularly in exclusively breastfed infants. Late HDN is still an important cause of mortality and morbidity in developing countries where vitamin $\mathrm{K}$ prophylaxis is not routinely practiced. Children with cholestatic liver disease are at risk for developing secondary vitamin $\mathrm{K}$ deficiency because of fat malabsorbtion and inadequate dietary intake. In this study, we described 11 infants (4 female, 7 male) with cholestatic liver disease with different etiologies exhibiting intracranial hemorrhage $(\mathrm{ICH})$. Six patients underwent surgical evacuation of $\mathrm{ICH}$, following the administration of vitamin $\mathrm{K}$ and / or fresh frozen plasma. The possibility of cholestatic liver disease should be considered in the treatment of $\mathrm{ICH}$ due to vitamin $\mathrm{K}$ deficiency.

\section{4}

\section{CHILDREN WITH SEVERE DEHYDRATION TREATED IN INTENSIVE CARE UNIT}

\section{Azemi}

\section{Peadiatric Department, University Children's Hospital, Prishtina, Serbia}

The object of study was to present children with severe dehydration caused by acute diarrhoea, due to the acute diarrhoea treated in Intensive Care Unit of the Paediatric Clinic.

The examinees were infants and preschool age children hospitalized on this ward during 2007.

The diagnosis was based on clinical assessment and laboratory tests.

During 2007, 657 children of different age were treated in Intensive Care Unit. Out of that number $462(70.3 \%)$ cases were infants, whereas only $42(6,3 \%)$ were preschool children. The highest number of them 196 (29.8\%) were cases with severe dehydration cased by acute diarrhoea syndrome; in addition to this number there were 187 cases $(27.7 \%)$ that dehydrated due to decompensate bronchopneumonia, whereas $12(1.8 \%)$ cases were patients with acute intracranial infections. With regard to the types of dehydration, there were $100(51.0 \%)$ cases with isonatremic dehydration, $54(27.55 \%)$ with hyponatremic dehydration and $42(21.42 \%)$ cases with hipernatemic dehydration. The lowest ph level was 6.80 , the excess of alkali $-30 \mathrm{mmol} / \mathrm{l}$, urea up to $18 \mathrm{mmol} / \mathrm{L}$. The rehydration has been administered on the basis of clinical assessment of dehydration level, correction of electrolyte disturbances, types of dehydration and correction of the metabolic acidosis and the antidiarrheal diet.

Severe dehydration still prevails as urgent condition in the Intensive Care Unit, being a medical and social issue.

Keywords: Severe dehydration

\section{5}

\section{SMALL FOR GESTATIONAL AGE NEWBORNS (SGA) AND COELIAC DISEASE: A CASE- CONTROL STUDY}

\author{
M.E. Baldassarre ${ }^{1}$, A. Laneve ${ }^{1}$, C. Longo ${ }^{1}$, \\ F. Manca ${ }^{2}$, N. Laforgia ${ }^{1}$
}

${ }^{1}$ DIGON, Section of Neonatology and NICU, ${ }^{2}$ Dipartimento di Scienze Storiche e Geografiche, Sezione di Statistica, University of Bari, Bari, Italy

A SGA birth could be a symptom of undiagnosed $C D$ in mother .Early diagnosis and treatment may avoid damages (1).

Aim: To determine the prevalence of untreated CD among SGA mothers (SgaM).

Methods: From September 2007 to October 2009, SgaM (cases) and Nga mothers (NgaM) were enrolled. Exclusion criteria: twin birth, artificial insemination. Blood samples were taken (5 cc) and antihuman $\lg A$ and $\lg G$ classes antitissue transglutaminase antibodies (IgA and IgG anti-tTg ab) were tested by ELISA commercial kit (Eurospital, Trieste, Italy); total IgA ab were tested. Statistical analysis: Fisher exact test, Odd ratio (OR).

Results: 193 SgaM (mean age 31,7 y) and 230 NgaM (mean age 31,1y) were enrolled. SGA newborns were like NGA newborns except for the for the body weight at birth $(1977 \pm 584,5 \mathrm{~g}$ vs. $2830 \pm 701,6 \mathrm{~g}$; $\mathrm{p}<0,05)$. SgaM had an higher risk of spontaneous 
abortions $(\mathrm{OR}=1,2)$. In studied population we found 3 celiac mothers $(0.7 \%)$ at gluten free diet (GFD); in $3 / 423(0,7 \%)$ woman IgA and IgG antitTg ab were positive:1/193 $(0,51 \%)$ was SgaM and $2 / 230(0,86 \%)$ were $\mathrm{NgaM}(\mathrm{p}=\mathrm{ns})$. In the new three cases an intestinal biopsy showed subtotal villous atrophy (Marsh 2) and HLA determination showed DQ2 positivity.

Conclusions: Undiagnosed CD doesn't seem more frequent among SGA mothers than in the general population. However, screening for CD during pregnancy could lead to the effective strategies for the prevention of such disease.

1) Hadziselimovic F, Geneto $R$, Buser M. Fetal Pediatr Pathol. 2007 May-Jun;26(3):125-34.

\section{6}

\section{ELEMENTAL FORMULA DIET IN AUTISTIC CHILDREN}

S. Karkelis, O. Papadaki-Papandreou, M. Lykogeorgou, T. Papandreou, L. Lianou, G. Chrousos

A' Department of Pediatrics, 'Agia Sofia' Children's Hospital, University of Athens, Athens, Greece

Object of study: Autism is a complexed neurodevelopmental-neurobiological disorder of behaviour. The aim of the study was the investigation of the correlation of the autistic children's hyperactivity status with major nutritional changes using elemental formula diet and testing these children for food allergies using Rast/Cap IgE tests.

Method: A total number of 45 children aged 2,5 to 8 years were checked. All of them were diagnosed with pervasive developmental disorder (international criteria 1994). From the 45 children, 17 were found having positive Cap IgE tests for cow milk allergy (class 1-3) and 3 of the 17 children had multinutritional allergy. Further, the 17 children group was randomly divided in two subgroups, 9 of the children started elemental formula(containing free aminoacids-Neocate ${ }^{\circledR}$ ) diet with exclusion of all milk products or milk containing food(group A) and the rest 8 children continued their previous diet(group B).The other 28 children group was also randomly divided in two subgroups, 13 children started elemental formula(containing free aminoacidsNeocate $\left.{ }^{\circledR}\right)$ diet with exclusion of all milk products or milk containing food(group C) and the rest 15 children continued their previous diet(group D).
Results: After 4 months of observation, statistical significant differences ( $p<0,001)$ were found in hyperactivity status(measured using properly arranged questionnaire model and special scoring system) between groups $A$ and $B$, also between groups $\mathrm{C}$ and $\mathrm{D}$.

Conclusions: Children suffering from pervasive developmental disorderhave increased hyperactivity status but it seems that elemental diet has much to offer.

787

\section{FECAL CALPROTECTIN IN AUTISTIC CHILDREN BEFORE AND AFTER THE USE OF ELEMENTAL DIET}

S. Karkelis, O. Papadaki-Papandreou, M. Lykogeorgou, T. Papandreou, L. Lianou, G. Chrousos

A' Department of Pediatrics, 'Agia Sofia' Children's Hospital, University of Athens, Athens, Greece

Object of study: Autism is a complexed neurodevelopmental-neurobiological disorder of behaviour. From the other hand, gastrointestinal problems like constipation, diarrhea or vomiting are often seen in autistic children and they are very difficult to resolve. The aim of the study was the investigation of the degree of possible inflammation of the autistic children's intestine mucosa by measurement of fecal calprotectin levels and observation of their changes after the introduction of elemental diet.

Method: A total number of 65 children aged 2,5 to 8 years were checked(group A). All of them were diagnosed with pervasive developmental disorder (international criteria 1994). Further, the children were randomly divided in two groups, 32 of the children started elemental formula(containing free aminoacids-Neocate ${ }^{\circledR}$ ) diet with exclusion of all milk products or milk containing food(group B) and the rest 33 children continued their previous diet(group C). Also, fecal calprotectin levels were measured in samples of 22 healthy children(aged 2 to 8 years) having normal diet(group D).

Results: Statistical significant differences $(p<0,001)$ were found in fecal calprotectin levels between groups A and D. Also, after 4 months of observation, statistical significant differences $(p<0,001)$ were found in fecal calprotectin levels between groups $B$ and $\mathrm{C}$. 